\title{
Análisis del liderazgo social de las pymes de Santa Marta - Colombia-, para el post-acuerdo desde el enfoque de la Responsabilidad Social Empresarial
}

Devinso Jiménez Sierra ${ }^{1}$ Corporación Unificada de Educación Superior C.U.N. Regional Magdalena Devinso_jimenez@cun.edu.co

Alejandrina Rincón Acevedo 2 Corporación Unificada de Educación Superior C.U.N. Regional Magdalena alejandrina_Acevedo@cun.edu.co

DOI: https://doi.org/10.21158/01208160.n85.2018.2051

Fecha de recepción: 13 de marzo de 2018

Fecha de aprobación: 11 de septiembre de 2018
Leonardo Pérez Suescún ${ }^{3}$ Universidad del Magdalena leopesu@gmail.com

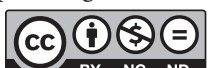

Cómo citar este artículo / To reference this article / Comment citer cet article / Para citar este artigo:

Jiménez Sierra, D.; Rincón Acevedo, A. y Pérez Suescún, L. (2018). Análisis del liderazgo social de las pymes de Santa Marta para el post-acuerdo desde el enfoque de la RSE. Revista EAN, 85, 105-124. DOI: https://doi.org/10.21158/01208160. n85.2018.2051

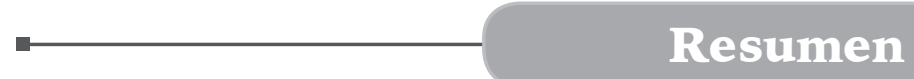

El artículo presenta un análisis correlacional entre los estilos de liderazgo propuestos por Kurt Lewin y la disposición para asumir acciones de Responsabilidad Social Empresarial en pymes de la ciudad de Santa Marta en el contexto del post-acuerdo. Como objetivo se estableció analizar el liderazgo social de las pymes para el post-acuerdo desde la perspectiva de la RSE, para ello en una primera fase, se aplicó el índice de liderazgo de las pymes por medio del test de liderazgo de Kurt Lewin estimando las variables género, cualificación y tamaño de las empresas. En una segunda fase se determinó el índice de disposición de las pymes para participar en procesos vinculados al post-acuerdo teniendo en cuenta aspectos como construcción para paz, inclusión, desarrollo económico y el reúso de insumos propios para la operación de las organizaciones. En una tercera fase se prueba la correlación entre el liderazgo y la disposición para participar en procesos vinculados a post-acuerdo.

\section{Palabras clave}

Liderazgo, pymes - Santa Marta-, pequeña y mediana empresa, responsabilidad social de los negocios, posconflicto, desarrollo económico.

Filósofo, Magister en Gerencia De Las Organizaciones de la Universidad del Zulia.

Maestrante en Administración gerencial en la Universidad Benito Juárez UBJ de la ciudad de México.

3 Contador Público. Administrador de Empresas. Especialista en Finanzas de la universidad del norte. Especialista en Administración De La Informática Educativa de la Universidad De Santander. Magíster En Desarrollo Sostenible y Medio Ambiente de la Universidad De Manizales. Doctorando En Ciencias Gerenciales de la Universidad Rafael Belloso Chacin, URBE. 


\section{Analysis of social leadership in pymes in Santa Marta - Colombia-, for the post- conflict agreement from the Corporate Social Responsibility perspective}

Abstract. This article shows a correlational analysis between leadership styles suggested by Kurt Lewin and the attitude towards performing corporate social responsibility actions in PyMES in the city of Santa Marta during the post- conflict agreement. It aims at analyzing the social leadership perspective in PyMES to face the post-conflict period under Corporate Social Responsibility. For this, in the first stage, a leadership ratio in PyMES is applied by using Kurt Lewin's leadership test, including variables such as gender, qualification and size of companies. Secondly, a disposition ratio in PyMES is determined to participate in post-conflict processes taking into account peace construction process, inclusion, economic development and the use of own supplies again in organizational operations. Next, the correlation between leadership and disposition is proved to participate in post-conflict agreement processes.

Key words. Leadership; pymes, Santa Marta, small and medium size companies, corporate social responsibility, post- conflict period, economic development.

\section{Analyse du leadership social des PME de Santa Marta —Colombie—, sous l'angle de la Responsabilité Sociale Entrepreneuriale dans le contexte du post-conflit}

Résumé. L'article présente une analyse corrélationnelle entre les styles de leadership proposés par Kurt Lewin et la prise de mesures de responsabilité sociale des PME de la ville de Santa Marta dans un contexte de post-conflit. L'objectif de cet article est d'analyser le leadership social des PME en cette période de post-conflit sous l'angle de la Responsabilité Sociale des Entreprises (RSE). Le test de leadership de Kurt Lewin a été appliqué à l'indice de leadership des PME lors d'une première phase d'analyses prenant en compte les variables de la qualification et du sexe des participants ainsi que la taille des entreprises. Lors d'une seconde phase d'analyses nous avons tenté de déterminer l'indice de volonté des PME à participer aux processus liés au post-conflit en prenant en compte des aspects tels que la construction de la paix, l'inclusion, le développement économique et la réutilisation des intrants dans le fonctionnement des organisations. Enfin, un test permettant de montrer la corrélation entre leadership et volonté de participation à des processus liés au post-conflit a été réalisé.

Mots clefs. Leadership, PME - Santa Marta, petites et moyennes entreprises, responsabilité sociale des entreprises, post-conflict, développement économique.

\section{Análise da liderança social das PMEs de Santa Marta —Colômbia—, para o pós-acordo desde o enfoque da Responsabilidade Social Empresarial}

Resumo. O artigo apresenta uma análise correlacional entre os estilos de liderança propostos por Kurt Lewin e a disposição para assumir ações de Responsabilidade Social Empresarial em PMEs da cidade de Santa Marta, no contexto do pós-acuerdo. Como objetivo se estabeleceu analisar a liderança social das PMEs para o pós-acordo desde a perspectiva da SER. Para isso numa primeira fase, aplicou-se o índice de liderança das PMEs por meio do teste de liderança de Kurt Lewin estimando as variáveis gênero, qualificação e tamanho das empresas. Numa segunda fase determinou-se o indice de disposição das PMEs para participar em processos vinculados ao pós-acordo considerando aspectos como a construção para a paz, a inclusão, o desenvolvimento econômico e a reutilização de insumos próprios para a operação das organizações. Numa terceira fase examina-se a correlação entre a liderança e a disposição para participar em processos vinculados ao pós-acordo.

Palavras-chave. Liderança, PMEs -- Santa Marta, pequena e média empresa, responsabilidade social dos negócios, pós-conflito, desenvolvimento econômico. 


\section{Introducción}

a firma del acuerdo de paz con las FARC
ha dado inicio a un escenario inédito en la historia del país, un contexto que requiere actores dispuestos a asumir un rol protagónico en el denominado post-acuerdo. Para ello debe contarse con el liderazgo del sector productivo más allá de la simple prescripción económica y legal. La participación proactiva de los líderes como una respuesta a soluciones puntuales de las necesidades de su entorno, aminorando las brechas e insuficiencias, morales, económicas y sociales del semejante (Barradas, 2013). Sin embargo, el logro de esta meta requiere conocer la manera en que las organizaciones predisponen sus liderazgos en favor de la realidad actual del país, en general, y de la ciudad, en particular.

El liderazgo social se entiende como la actuación éticamente responsable frente a la construcción de un modelo económico fundado en la sustentabilidad y el bienestar de los grupos de interés de la organización. Esta postura requiere de la planificación de metas ajustadas al compromiso social que exige el estado actual de un mundo con recursos limitados, con una amplia tradición de consumo salvaje y sin control. Un líder social se prepara para que su organización no sucumba ante presiones externas estandarizadas que propenden por una economía de mercados sin control ni responsabilidad (Ospina, 2006).

Dentro de esta perspectiva, los gerentes líderes, con visión social, estiman los impactos de la organización asociados al medio ambiente, mejorando la operación y sus derivados con un enfoque sostenible (Radrigán, Dávila y Penaglia, 2012). En Colombia, y en particular Santa Marta, una ciudad con potencialidades competitivas desde lo ambiental y en medio de un escenario de post-acuerdo, requiere de un compromiso apremiante para cimentar valores, prácticas y acciones hacia la Responsabilidad Social Empresarial.

Además, los postulados que describen teóricamente los stakeholders reconocen el valor redistributivo del poder en las organizaciones. Este ejercicio en el que cada grupo de interés tiene preponderancia y un rol en el desempeño final, genera sinergias que requieren la filiación gerencial de un liderazgo social. Al combinar una disposición de las organizaciones de modo responsable con la actuación de una gerencia social desde el liderazgo participativo, obtenemos compromisos fiables enmarcados en la responsabilidad empresarial (Gandica, 2008). Por ello, es importante reconocer que el contexto actual del post-acuerdo con las FARC representa una oportunidad social para crear espacios de integración con esta masa crítica de ciudadanos. Para ello la disposición y el liderazgo son esenciales.

Dentro de los perfiles de liderazgos el que mayor acercamiento tiene con los aspectos sociales de la organización es el perfil democrático, este permite la adhesión de nuevos conceptos prescritos por el contexto interno y externo de la organización, lo que puede generar una mayor disposición para incorporar políticas, acciones, prácticas o planes de responsabilidad social (Kliksberg, 2009). Dentro de esta disposición podríamos ubicar el fenómeno social llamado post-acuerdo en el marco político colombiano. Es decir, un perfil de liderazgo democrático facilitaría la participación de la organización en aspectos asociados a la realidad de la región, en particular de Santa Marta.

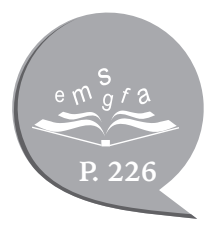


La pregunta que se hace a través del desarrollo de la investigación es: ¿Sí existe un liderazgo «social» que permita generar una disposición de las organizaciones para el post-acuerdo? El contexto de la investigación nos permitió formular como objetivo de la investigación: analizar el liderazgo social de las pymes para el post-acuerdo desde la perspectiva de la RSE. Esta problemática se aborda desde dos perspectivas, primero, la identificación de los perfiles de liderazgo de las organizaciones objeto de estudio desde el enfoque participativo y transformacional. Segundo, la disposición de las pymes de la ciudad de Santa Marta para el post-acuerdo, pero desde una mirada de la Responsabilidad Social Empresarial, entendiendo que esta necesidad de integración participativa constituye una responsabilidad que el líder debe asumir como aporte y retribución a su bonanza económica (Barradas, 2013).

\section{Fundamento teórico}

$\mathbf{E}^{x}$ xisten diferentes estilos que proponen funciones, roles y actividades que deben desarrollar los líderes y que dan vía a varios enfoques de liderazgo, desde los postulados de la universidad de Ohio y Harvard, pasando por la malla gerencial de Blake y Mounton, los modelos de liderazgo participativos de VroomYetton y Kurt Lewin, los liderazgos situacionales de Blanchard, el liderazgo transformacional de Bass, hasta las tendencias de liderazgo por competencia (Goleman, 2016).

El artículo aborda el enfoque participativo del liderazgo de Kurt Lewin en el que se discriminan tres estilos de liderazgo que se adaptan a las condiciones de las organizaciones de la ciudad de Santa Marta, caracterizadas por su escasa dinámica organizacional, pocos niveles jerárquicos y orientados en las metas más que en las personas: autoritario, democrático y laissez faire. Según Lewin en el estilo de liderazgo autoritario no hay participación directa del grupo de trabajo, las directrices misionales $\mathrm{y}$ visionales responden exclusivamente a la gerencia sin advenimiento de las iniciativas o percepciones de los colaboradores, el líder concentra todo el poder y la toma de decisiones. En un segundo estilo, el democrático, las directrices del líder son debatidas por el grupo y decididas por su estímulo y apoyo.
Se basa en la colaboración y participación de todos los miembros del grupo. El líder y los subordinados actúan como una unidad. Un tercer estilo es el laissez faire, es una expresión francesa que significa dejen hacer o dejen pasar. De ahí que este estilo de liderazgo se caracterice por una libertad completa por parte del grupo en las decisiones y una participación mínima del líder, este no ejerce su función, no se responsabiliza del grupo y lo deja a su propia iniciativa. Aunque algunos estudios han mostrado la importancia del empleo de diferentes estilos en los que predominan en especial el autoritario, el democrático y el laissez faire (Goleman, 2015).

Aunque los postulados de Lewin se sitúan en el espectro de los modelos participativos, es importante reconocer que sus fundamentaciones apuntan a la trasformación de los equipos de trabajo, un cambio de carácter como se denominó, posteriormente, en el liderazgo transformacional. En su teoría de campo propuso que el contexto y el espacio tiempo presente ejerce un influjo sobre cualquier objeto que se halla en él. Este efecto campo se evidencia tanto en la conducta individual como en la dinámica grupal. En relación con la dinámica de grupo, sostuvo que el análisis de campo constituía una herramienta básica 
para determinar la posición relativa de sus componentes, la estructura del grupo y su situación en el entorno (Fernández y Puentes, 2014). Para Lewin, la teoría de "campo" se apoya en el supuesto de que el campo psicológico de los individuos se organiza en conformidad con leyes universales que permiten esquematizar los cambios que se producen en los individuos y grupos; sus causas y efectos (Lewin, 1991).

En el liderazgo transformacional se busca generar una situación de cambio, un movimiento de carácter que permita, como lo afirmaba Kurt Lewin, descongelamiento del carácter del seguidor para asumir una nueva acción, lo que genera un movimiento hacia el logro y una nueva adaptación hacia el recongelamiento del carácter del colaborador (Hesselbein, 2010).

Es importante identificar dos enfoques que existen dentro de la fundamentación teórica de lo transformacional: los conceptos de «transaccional» $y$ «transformador». Los líderes transaccionales se centran en la acción, proponen una relación de intercambio entre la fuerza de trabajo que entrega el colaborador y el requerimiento de la organización; una acción del colaborador por una meta propuesta por el líder. El liderazgo trasformador tiene su enfoque en la visión general y desde allí determina la acción ajustada a la meta (Bennis, 2001).

El líder transaccional se plantea tres escenarios posibles: a) recompensa contingente, b) administración por excepción activa: el líder busca desviaciones de las reglas y normas tomando acciones correctivas, c) administración por excepción pasiva: el líder interviene solo si no se satisfacen las normas, asumiendo un estilo laissez faire, asume sus responsabilidades y evita tomar decisiones (Acosta, 2015).

El liderazgo transformador, por su parte, reconoce y explota una necesidad o demanda personal existente de un seguidor potencial. Se basa en la motivación potencial del seguidor para que este generé un cambio de carácter que le permita moverse, dirigirse hacia donde potencialmente el líder le requiere. El resultado de este liderazgo es una combinación entre estimulo potencialidad, lo que genera una capacidad mayor orientada a valoraciones axiológicas. Al final el seguidor se hace consciente de su movimiento y cambio de carácter (Pírela, Camacho y Sánchez, 2004). 


\section{Metodología}

L a investigación es de tipo correlacional dy enfoque cuantitativo, se establecieron niveles de liderazgo por medio del test de perfiles de liderazgo de Kurt Lewin, convirtiendo los niveles en escalas representadas en un mismo número de índices para estimar nuestra primera variable, «índice de niveles de liderazgo de las pymes de la ciudad de Santa Marta». Esta variable se cruzó con la variable «disposición de las pymes de la ciudad de Santa Marta para el post-acuerdo", para determinar la correlación entre variables probando la aceptación o negación de la hipótesis calculando el chi-cuadrado entre ambas variables.

En una primera fase se realiza una validación de la base de datos de la cámara de comercio de la ciudad de Santa Marta para determinar los criterios de selección de la población objeto de estudio, en este caso se determinan los establecimientos de comercio pequeños, medianos (tabla 1) con registro mercantil vigente a corte de julio de 2016 . Luego se realizó la contextualización del test de niveles de liderazgo de Kurt Lewin que consta de una batería de 33 ítems con un coeficiente alfa Cronbach de 0.81345 mostrando una validación suficiente.
En una segunda fase se diseñó un instrumento para medir el grado de disposición de las pymes de Santa Marta para el post-acuerdo, para diseñarlo se utilizó un árbol de secuencia: variable/dimensión/indicador/ítems, y se tuvieron en cuenta factores encontrados en el análisis de los antecedentes: desarrollo socioeconómico/gestión/estrategia para la paz/participación/capital humano/gestión ambiental. La primera batería de indicadores arrojo 37 ítems distribuidos en 4 dimensiones, se aplicó una validación de constructo y contenido por vía de jueces expertos lo que redujo la batería a 20 ítems con los que se aplicó la prueba piloto y se midió su confiabilidad con el coeficiente de alfa Cronbach: 0.85 (figura 1). La validez de constructo «se refiere a qué tan exitosamente un instrumento representa y mide un concepto teórico» (Hernández, 2006, p. 197). En una tercera fase se probó la hipótesis de la investigación con el coeficiente chi-cuadrado, formulada así:

$$
\mathrm{H}_{\mathrm{o}} \rightarrow \mathrm{X}_{\mathrm{nb}}=\mathrm{X}_{\mathrm{na}} \mathrm{y} / \mathrm{o} \mathrm{H}_{1} \rightarrow \mathrm{X}_{\mathrm{nb}} \neq \mathrm{X}_{\mathrm{na}} \text {. }
$$

Figura 1. Alfa de Cronbach - Consistencia interna del instrumento de medición

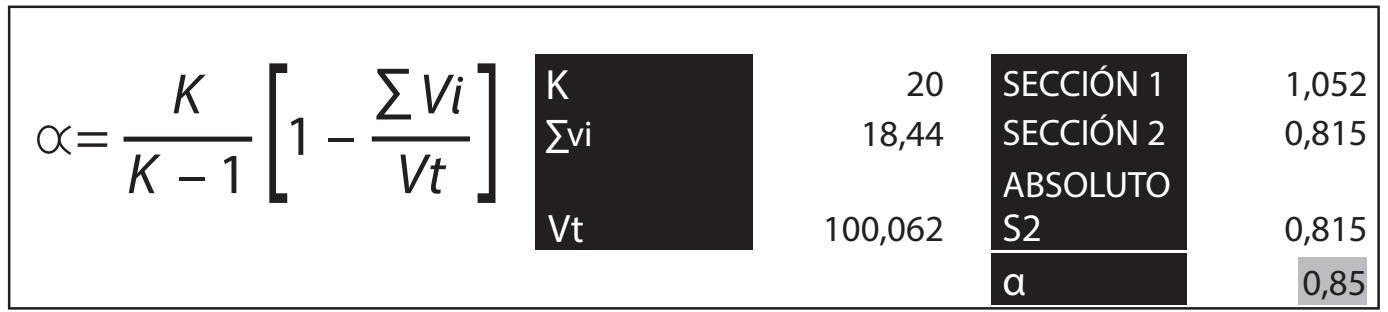

Fuente. Elaboración propia. 
Tabla 1. Población y muestra

\begin{tabular}{|l|l|c|c|}
\hline \multicolumn{4}{|c|}{ Distribución de población y muestra } \\
\hline Tamaño de empresa & Clasificación según Ley 905/2004 & Universo & Muestra \\
\hline Empresas pequeñas & Entre 11 y 50 empleados & 170 & 160 \\
\hline Empresas medianas & Entre 51 y 200 empleados & 34 & 34 \\
\hline Total pymes Santa Marta & & 204 & 194 \\
\hline
\end{tabular}

Fuente. Elaboración propia con base en de datos de la Cámara de Comercio de Santa Marta, corte 2016.

Tabla 2. Ficha técnica cálculo de muestreo

\begin{tabular}{|l|}
\hline \multicolumn{1}{|c|}{ Ficha técnica } \\
\hline Universo: 204 establecimientos de comercio \\
\hline Margen de error: $3 \%$ \\
\hline Nivel de Confianza: $97 \%$ \\
\hline Tamaño de la muestra: 194 establecimientos de comercio \\
\hline
\end{tabular}

Fuente. Cálculo electrónico con la plataforma Adimen, 2017.

Tabla 3. Fases de la investigación

\begin{tabular}{|c|l|}
\hline \multicolumn{2}{|c|}{ FASES DE LA INVESTIGACIÓN } \\
\hline Fase 1 & Aplicación del test de liderazgo de Kurt Lewin: índice de liderazgo: variable 1 \\
\hline Fase 2 & $\begin{array}{l}\text { Diseño, validez y medición de la confiabilidad del instrumento: disposición de } \\
\text { pymes para el posconflicto: variable } 2\end{array}$ \\
\hline Fase 3 & Análisis de resultados y prueba de hipótesis \\
\hline
\end{tabular}

Fuente. Elaboración propia, 


\section{Resultados}

\subsection{Resultados fase 1}

En una primera fase, como consecuencia del logro del objetivo número uno, se aplicó el test de Kurt Lewin a 160 empresas pequeñas y 34 medianas de la ciudad de Santa Marta, el test consta de una batería de 33 ítems que clasifica los resultados en una escala de 0.33 a 1 , determinando un rango de valor distribuido en los tres tipos de liderazgo que propone el test. La tabla 4 y la figura 2 muestran que las pymes tienen un índice de liderazgo de 0.74 , que lo sitúa en el cuartil más bajo del perfil de liderazgo democrático. Lo que indica que la media aritmética de la muestra tiende a la gerencia desde un perfil democrático, lo que auspicia la participación, la generación de ideas generando un entorno incluyente lo que podría favorecer una eventual proposición de acciones de responsabilidad social en las pequeñas y medianas empresas.

Tabla 4. Estadísticos de la variable: índice de liderazgo

\begin{tabular}{|c|c|c|c|c|c|c|c|}
\hline & $\mathrm{N}$ & Mínimo & Máximo & Media & Desv. típ. & \multicolumn{2}{c|}{ Asimetría } \\
\cline { 2 - 8 } & Estadístico & Estadístico & Estadístico & Estadístico & Estadístico & Estadístico & Error típico \\
\hline VAR00005 & 194 & .33 & 1.00 & 0.7499 & .18454 & .104 & .175 \\
\hline $\begin{array}{c}\text { N válido } \\
\text { (según lista) }\end{array}$ & 194 & & & & & & \\
\hline
\end{tabular}

Fuente. Elaboración propia desde SPSS V. 21, 2017.

Figura 2. Índice de liderazgo

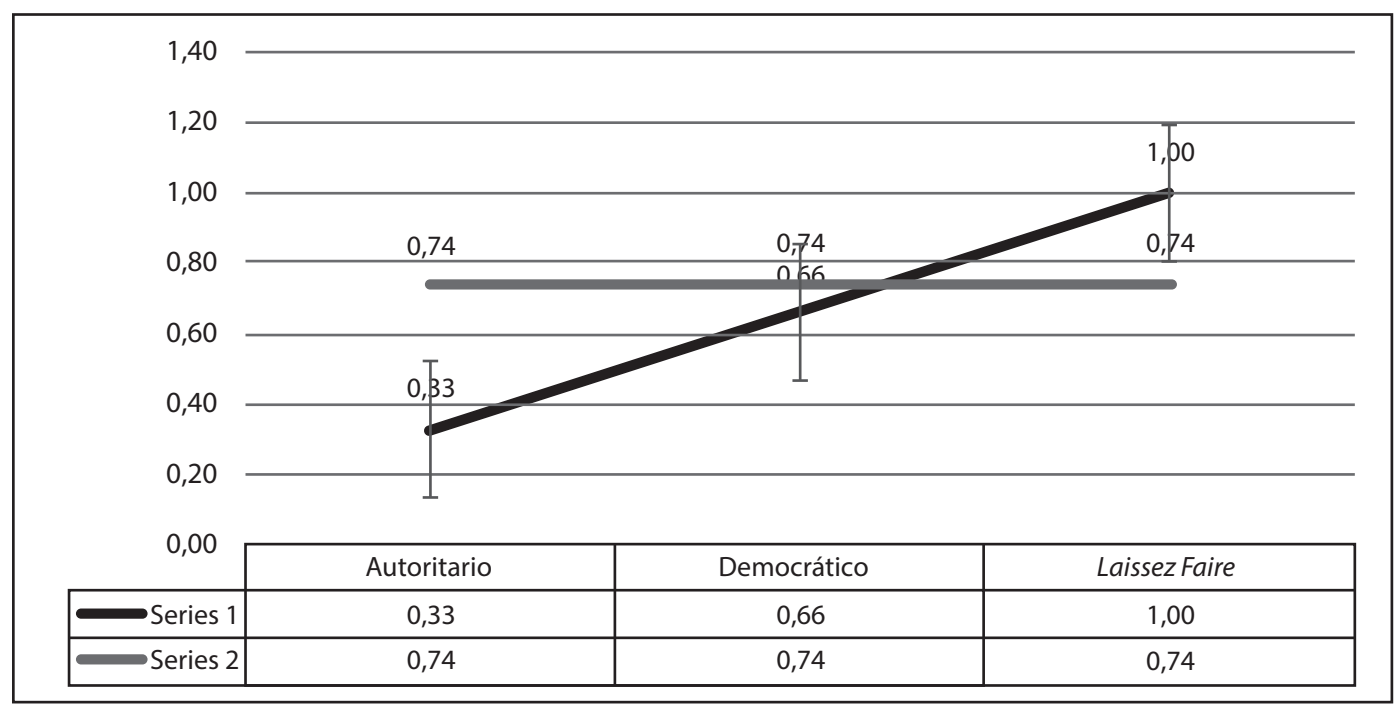

Fuente. Elaboración propia.. 
La tabla 5 y la figura 3 muestran la distribución de los perfiles de liderazgo en los que se puede apreciar que, aunque la media de las empresas está en el índice de liderazgo democrático con $63 \%$, existe una participación del $31 \%$ que se inclinan por un liderazgo laissez faire, y un $5 \%$ de índice de liderazgo autoritario, lo que muestra que el indicador tiene una tendencia baja hacia el perfil de liderazgo laissez.

Tabla 5. Distribución de la variable: índice de liderazgo

\begin{tabular}{|c|c|c|c|c|c|}
\hline \multicolumn{2}{|c|}{} & Frecuencia & Porcentaje & Porcentaje válido & Porcentaje acumulado \\
\hline \multirow{3}{*}{ Válidos } & Autoritario & 10 & 5.2 & 5.2 & 5.2 \\
\cline { 2 - 6 } & Democrático & 123 & 63.4 & 63.4 & 68.6 \\
\cline { 2 - 6 } & Laissez faire & 61 & 31.4 & 31.4 & 100 \\
\cline { 2 - 6 } & Total & 194 & 100 & 100 & \\
\hline
\end{tabular}

Fuente. Jiménez y Rincón, 2017.

Figura 3. Distribución de la variable: índice de liderazgo

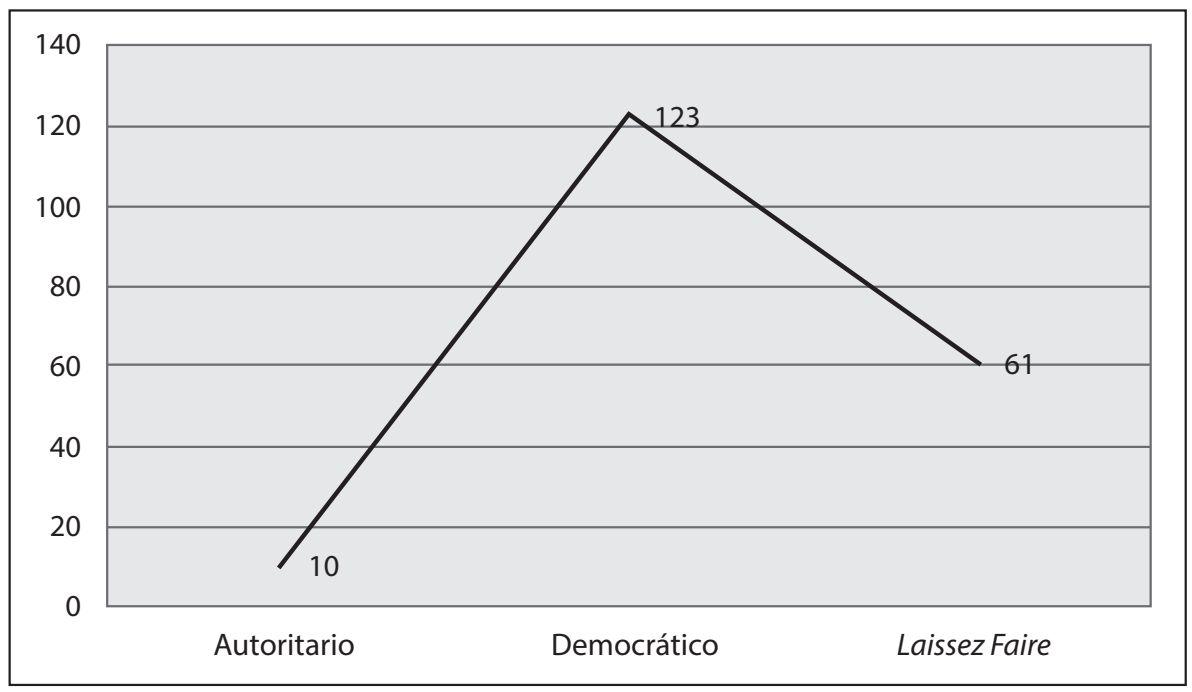

Fuente. Jiménez y Rincón, 2017.

La tabla 6 y la figura 4 muestran la relación que existe entre el tamaño de la empresa y su perfil de liderazgo, el resultado muestra que se mantiene el índice de liderazgo sobre el perfil democrático en las pequeñas empresas -de aquí en adelante PE- el perfil laissez faire alcanza un $27 \%$ sobre el total de toda la muestra de este estrato, con una participación de 54 empresas sobre una participación del 51.5 de PE que están en el perfil de liderazgo democrático, y un $3 \%$ en el perfil autoritario. En las empresas medianas -de aquí en adelante EM- la tendencia al perfil democrático tiene mayor demarcación con un $21 \%$ sobre el 7 $\%$ del perfil laissez faire, y un $2.1 \%$ en el perfil autoritario. 
Tabla 6. Contingencia de la variable índice de liderazgo vs. tipo de empresa

\begin{tabular}{|c|c|c|c|c|c|c|}
\hline & & & \multicolumn{3}{|c|}{ VAR00005 } & \multirow{2}{*}{ Total } \\
\hline & & & Autoritario & Democrático & Laissez faire & \\
\hline \multirow{4}{*}{ VAR00001 } & \multirow{2}{*}{$\mathrm{ME}$} & Recuento & 4 & 23 & 7 & 34 \\
\hline & & $\%$ del total & $2.1 \%$ & $11.9 \%$ & $3.6 \%$ & $17.5 \%$ \\
\hline & \multirow{2}{*}{ PE } & Recuento & 6 & 100 & 54 & 160 \\
\hline & & $\%$ del total & $3.1 \%$ & $51.5 \%$ & $27.8 \%$ & $82.5 \%$ \\
\hline \multirow{2}{*}{\multicolumn{2}{|c|}{ Total }} & Recuento & 10 & 123 & 61 & 194 \\
\hline & & $\%$ del total & $5.2 \%$ & $63.4 \%$ & $31.4 \%$ & $100 \%$ \\
\hline
\end{tabular}

Fuente: Jiménez y Rincón, 2017.

Figura 4. Contingencia de la variable: índice de liderazgo vs. tipo de empresa

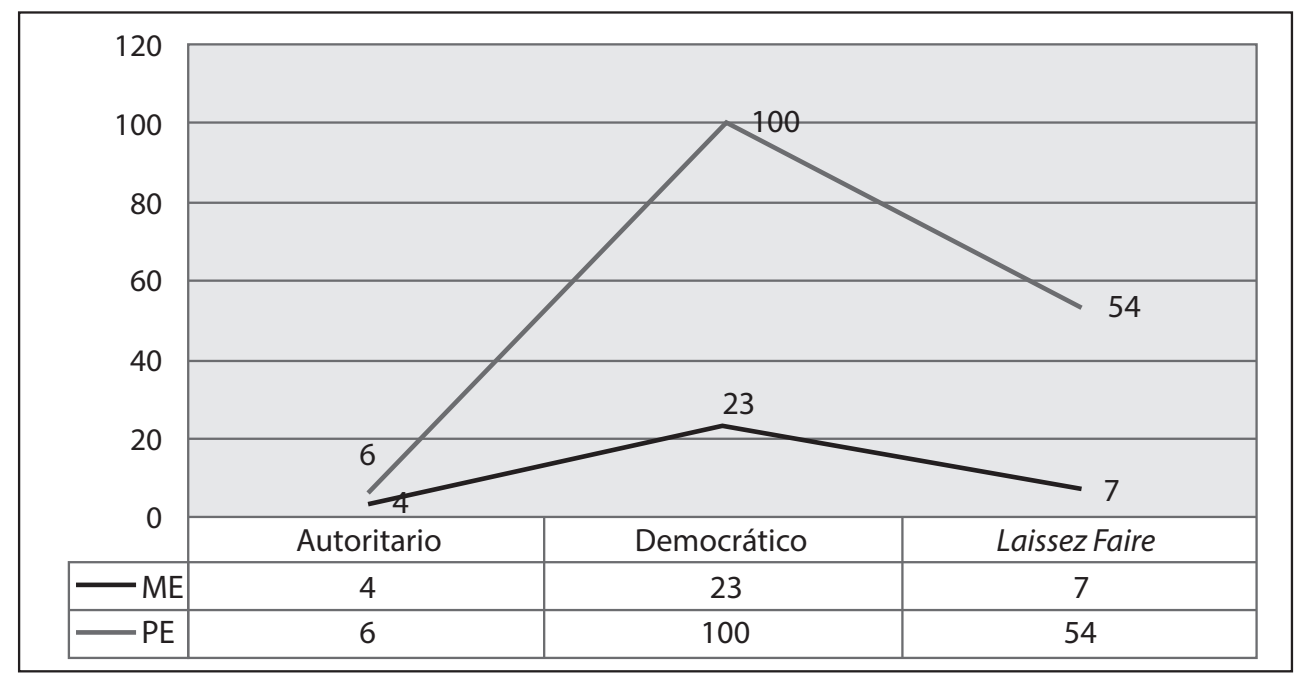

Fuente. Jiménez y Rincón, 2017.

La tabla 7 y la figura 5 muestran el comportamiento de los perfiles de liderazgo en razón del género, lo que arrojó proporciones casi idénticas; la diferencia entre los hombres y las mujeres que presentan perfil democrático es de solo 5 unidades, 64 de los hombres tienen perfil democrático frente a 59 de las mujeres.
En cuanto al perfil laissez faire la diferencia son 3 unidades; 32 mujeres y 29 hombres, donde más se presentan diferencias en el perfil autoritario donde las mujeres parecen ser más autoritarias que los hombres en una relación de 9 a 1. 
Tabla 7. Contingencia de la variable índice de liderazgo vs. género

\begin{tabular}{|c|c|c|c|c|c|c|}
\hline & & & \multicolumn{3}{|c|}{ VAR00005 } & \multirow{2}{*}{ Total } \\
\hline & & & Autoritario & Democrático & Laissez faire & \\
\hline \multirow{4}{*}{ VAR00002 } & \multirow{2}{*}{ Hombre } & Recuento & 1 & 64 & 29 & 94 \\
\hline & & $\%$ del total & $.5 \%$ & $33.0 \%$ & $14.9 \%$ & $48.5 \%$ \\
\hline & \multirow{2}{*}{ Mujer } & Recuento & 9 & 59 & 32 & 100 \\
\hline & & $\%$ del total & $4.6 \%$ & $30.4 \%$ & $16.5 \%$ & $51.5 \%$ \\
\hline \multirow{2}{*}{\multicolumn{2}{|c|}{ Total }} & Recuento & 10 & 123 & 61 & 194 \\
\hline & & $\%$ del total & $5.2 \%$ & $63.4 \%$ & $31.4 \%$ & $100 \%$ \\
\hline
\end{tabular}

Fuente. Jiménez y Rincón, 2017.

Figura 5. Contingencia de la variable: índice de liderazgo vs. género

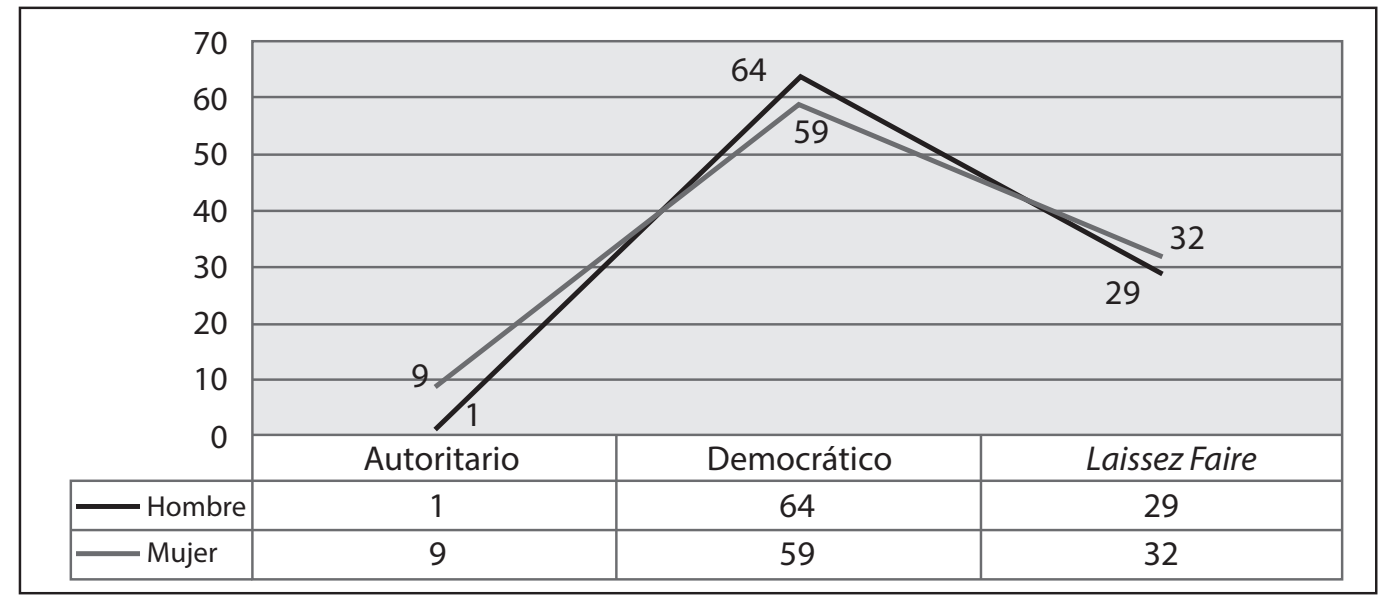

Fuente. Jiménez y Rincón, 2017.

Al cruzar la variable índice de liderazgo con la cualificación de las unidades de análisis en la tabla 8 y figura 6 se evidenció que la mayoría son profesionales de los cuales 78 tienen perfil democrático, 37 perfil laissez faire y 1 autoritario y representan el $58.8 \%$ de la muestra. Se siguen en porcentaje de participación los especialistas con un $20 \%$ de los cuales 20 tienen perfil laissez faire, 16 en perfil democrático y 4 en perfil autoritario. El tercer nivel de cualificación más recurrente es el de bachiller con una participación del $14.4 \%$ con 25 en perfil democrático, 3 en perfil laissez faire y 0 autoritario. El nivel de cualificación con menos participación es el de magíster con una participación del $5 \%$ con 5 en el perfil autoritario, 4 en el perfil democrático y 1 en el perfil laissez faire. Debe anotarse que, aunque se midió en el ejercicio la existencia de doctores este arrojó un $0 \%$ de participación. 
Tabla 8. Contingencia de la variable índice de liderazgo vs.cualificación

\begin{tabular}{|c|c|c|c|c|c|c|}
\hline & & & \multicolumn{3}{|c|}{ VAR00005 } & \multirow{2}{*}{ Total } \\
\hline & & & Autoritario & Democrático & Laissez faire & \\
\hline \multirow{8}{*}{ VAR00004 } & \multirow{2}{*}{ Bachillerato } & Recuento & 0 & 25 & 3 & 28 \\
\hline & & $\%$ del total & $0.0 \%$ & $12.9 \%$ & $1.5 \%$ & $14.4 \%$ \\
\hline & \multirow{2}{*}{ Especialista } & Recuento & 4 & 16 & 20 & 40 \\
\hline & & $\%$ del total & $2.1 \%$ & $8.2 \%$ & $10.3 \%$ & $20.6 \%$ \\
\hline & \multirow{2}{*}{ Magíster } & Recuento & 5 & 4 & 1 & 10 \\
\hline & & $\%$ del total & $2.6 \%$ & $2.1 \%$ & $.5 \%$ & $5.2 \%$ \\
\hline & \multirow{2}{*}{ Profesional } & Recuento & 1 & 78 & 37 & 116 \\
\hline & & $\%$ del total & $.5 \%$ & $40.2 \%$ & $19.1 \%$ & $59.8 \%$ \\
\hline \multirow{2}{*}{\multicolumn{2}{|c|}{ Total }} & Recuento & 10 & 123 & 61 & 194 \\
\hline & & $\%$ del total & $5.2 \%$ & $63.4 \%$ & $31.4 \%$ & $100 \%$ \\
\hline
\end{tabular}

Fuente. Jiménez y Rincón, 2017.

Figura 6. Contingencia de la variable: índice de liderazgo vs. cualificación

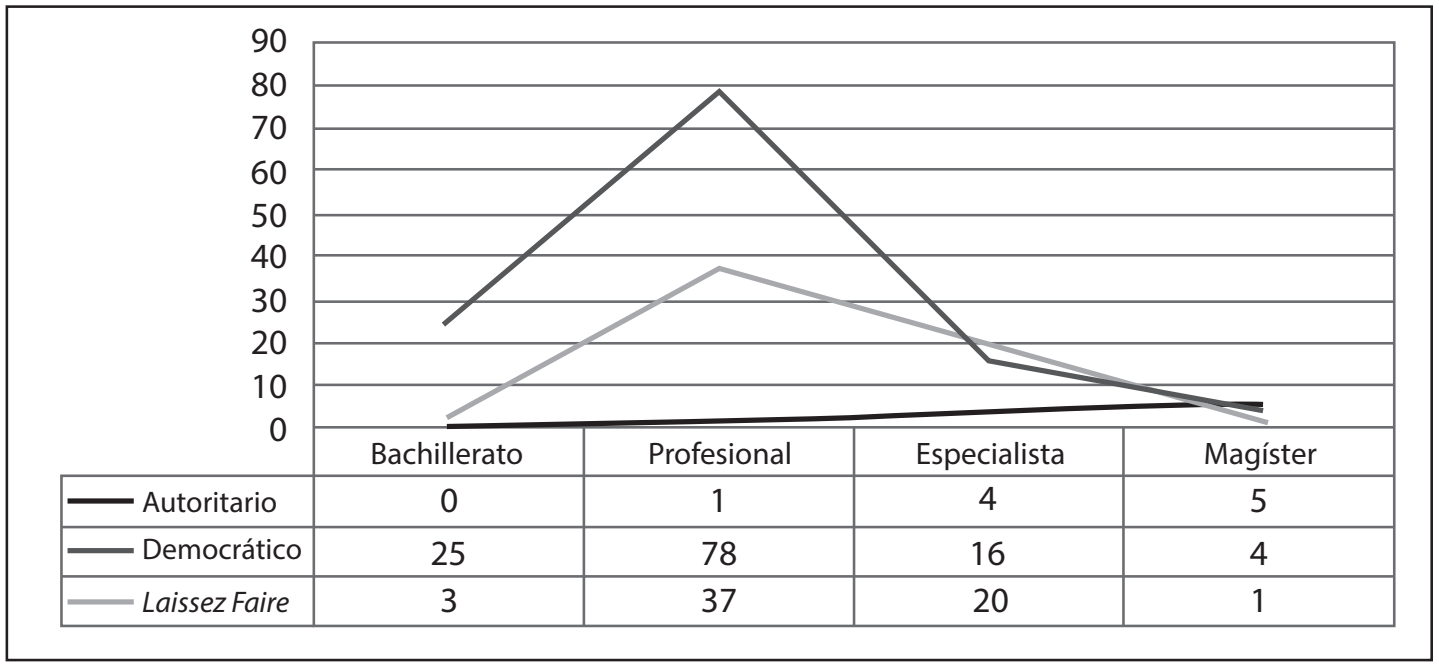

Fuente. Jiménez y Rincón, 2017. 


\subsection{Resultados fase 2}

En una segunda fase, para cumplir el objetivo específico número dos, se estableció un índice de significancia de la disposición de las pymes de Santa Marta frente al proceso de post-acuerdo que el gobierno desarrolla con las FARC desde elementos asociados a la Responsabilidad Social Empresarial (RSE). Para ello, se definió un baremo para determinar los índices de significancia en una escala tripartita tal como se detalla a continuación.

La tabla 9 y 10 muestran la distribución de las dimensiones en relación con su número de ítems, a saber, se exploran 4 dimensiones: una dimensión social con 9 ítems, dimensión económica con 3 ítems, dimensión ambiental con 4 ítems y una dimensión de participación con 4 ítems. Para determinar el índice de disposición de las pymes para el post-acuerdo en cada una de las dimensiones y en total para la variable se utiliza una escala de base (1), tal como lo muestra la distribución de la tabla número 8 . La escala que se utiliza contiene tres niveles de significancia: significativo (S), aceptablemente significativo (A_S_) y poco significativo (P_S_), que buscan determinar índices de disposición a nivel del ítem, la dimensión, y por supuesto, la variable de estudio.

Tabla 9. Dimensiones de la variable 2: disposición de pymes para el post-acuerdo

\begin{tabular}{|c|c|c|c|}
\hline \multirow{2}{*}{$\begin{array}{c}\text { N. } \\
\text { Dimensiones }\end{array}$} & DIMENSIONES & \multicolumn{2}{|c|}{ ÍTEMS } \\
\cline { 3 - 4 } & & NÚMERO DE ÍTEMS & ÍTEMS \\
\hline 1 & Dimensión social & 9 & $1-9$ \\
\hline 2 & Dimensión económica & 3 & $10-12$ \\
\hline 3 & Dimensión ambiental & 4 & $13-16$ \\
\hline 4 & Dimensión de participación & 4 & $17-20$ \\
\hline
\end{tabular}

Fuente. Jiménez y Rincón, 2017.

Tabla 10. Niveles de significancia: disposición de pymes para el post-acuerdo

\begin{tabular}{|l|c|}
\hline Nivel de significancia & Escala \\
\hline Significativo: S_ & $0,8 \geq 1$ \\
\hline Aceptablemente Significativo: A_S_ & $0,5 \geq 7,9$ \\
\hline Poco Significativo: P_S_ & $0 \geq 0,49$ \\
\hline
\end{tabular}

Fuente. Jiménez y Rincón, 2017. 
La tabla 11 y la figura 7 muestran el índice de disposición de la pymes para el post-acuerdo en relación con cada ítem, allí puede identificarse que 7 de los 20 ítems están en un nivel poco aceptable de disposición, ellos son los ítems: $1,5,6,9,12,13$ y 14. El ítem_1 con un índice de 0.4 y nivel poco significativo muestra la escasa disposición para la empleabilidad que tienen las pymes con personas que presentan discapacidad, lo que podría ser una dificultad al momento de participación en política pública para el post-acuerdo, entendiendo que según el censo que se realizó a los desmovilizados, la población con algún tipo de discapacidad supera el 30 \% (UNAL, 2017). El ítem_5 con un índice de 0.42 muestra la incipiente actividad de voluntariados de las pymes de Santa Marta. El ítem_6 y el ítem_9, relacionados con acciones en la construcción de paz, tienen un índice de 0.44 y 0.43 respectivamente, lo que muestra el poco interés en el post-acuerdo en términos productivos. El ítem_12 con un índice de 0.4 muestra la poca interrelación que tienen las pymes de Santa Marta con el mercado regional. El ítem_13 y 14, dan cuenta de la poca planeación en el ejercicio medioambiental, claves en el proceso de una visión de mundo sostenible y sustentable del sector productivo. Es importante resaltar que a pesar de que los demás ítems están situados en un nivel medio «aceptablemente significativo", se ubican en el cuartil más bajo del nivel, lo que en realidad es una deficiencia. Ítems como los que hacen relación al conocimiento sobre el documento de los acuerdos de paz con las FARC, aunque su índice es medio, no es contundente.

Tabla 11. Niveles de significancia de ítems de la variable 2: disposición de pymes para el post-acuerdo

\begin{tabular}{|c|l|c|c|c|}
\hline N. ${ }^{\prime}$ ítems & \multicolumn{1}{|c|}{ Ítems } & índice & $\begin{array}{c}\text { Nivel de } \\
\text { significancia }\end{array}$ & Cuartil \\
\hline ÍTEM_1 & $\begin{array}{l}\text { Incorporación de la fuerza de trabajo de personas en riesgo } \\
\text { de exclusión }\end{array}$ & 0.41 & P_S_ & 4 \\
\hline ÍTEM_2 & $\begin{array}{l}\text { Incorporación de la fuerza de trabajo de personas en } \\
\text { condición de discapacidad física }\end{array}$ & 0.52 & A_S_ & 1 \\
\hline ÍTEM_3 & Índice de empleabilidad & 0.58 & A_S_ & 1 \\
\hline ÍTEM_4 & Participación en proyectos sociales & 0.56 & A_S_ & 1 \\
\hline ÍTEM_5 & Participación en actividades de voluntariado & 0.42 & P_S_ & 4 \\
\hline ÍTEM_6 & Compromiso con la construcción de paz & 0.44 & P_S_ & 4 \\
\hline ÍTEM_7 & Participa en la construcción de paz & 0.56 & A_S_ & 1 \\
\hline ÍTEM_8 & $\begin{array}{l}\text { Conocimientos asociados a la firma del acuerdo con las } \\
\text { FARC }\end{array}$ & 0.57 & A_S_ & 1 \\
\hline ÍTEM_9 & $\begin{array}{l}\text { Acciones o prácticas relacionadas con el post-acuerdo con las } \\
\text { FARC }\end{array}$ & 0.43 & P_S_ & 4 \\
\hline ÍTEM_12 & Fomento de relacionamiento regional & El respeto a los derechos de los consumidores & 0.59 & A_S_ \\
\hline
\end{tabular}


Tabla 11. Niveles de significancia de ítems de la variable 2: disposición de pymes para el post-acuerdo (Continuación)

\begin{tabular}{|l|l|c|c|c|}
\hline ÍTEM_13 & Consumo eficiente de energía & 0.48 & P_S_ & 4 \\
\hline ÍTEM_14 & Planificación de impactos medioambientales & 0.44 & P_S_ & 4 \\
\hline ÍTEM_15 & Valoración del uso de envases y embalajes reciclables & 0.59 & A_S_ & 1 \\
\hline ÍTEM_16 & Uso de envases y embalajes reciclables & 0.58 & A_S_ & 1 \\
\hline ÍTEM_17 & Incorporación de fuerza de trabajo en proceso de reinserción & 0.60 & A_S_ & 1 \\
\hline ÍTEM_18 & Incorporación de fuerza de venta con enfoque inclusivo & 0.58 & A_S_ & 1 \\
\hline ÍTEM_19 & Certificación de ISO 26.000 & 0.56 & A_S_ & 1 \\
\hline ÍTEM_20 & $\begin{array}{l}\text { Incorporación de proyectos del gobierno para la construcción } \\
\text { de paz }\end{array}$ & 0.57 & A_S_ & 1 \\
\hline
\end{tabular}

Fuente. Jiménez y Rincón, 2017.

Figura 7. Niveles de significancia de ítems de la variable 2: disposición de pymes para el post-acuerdo

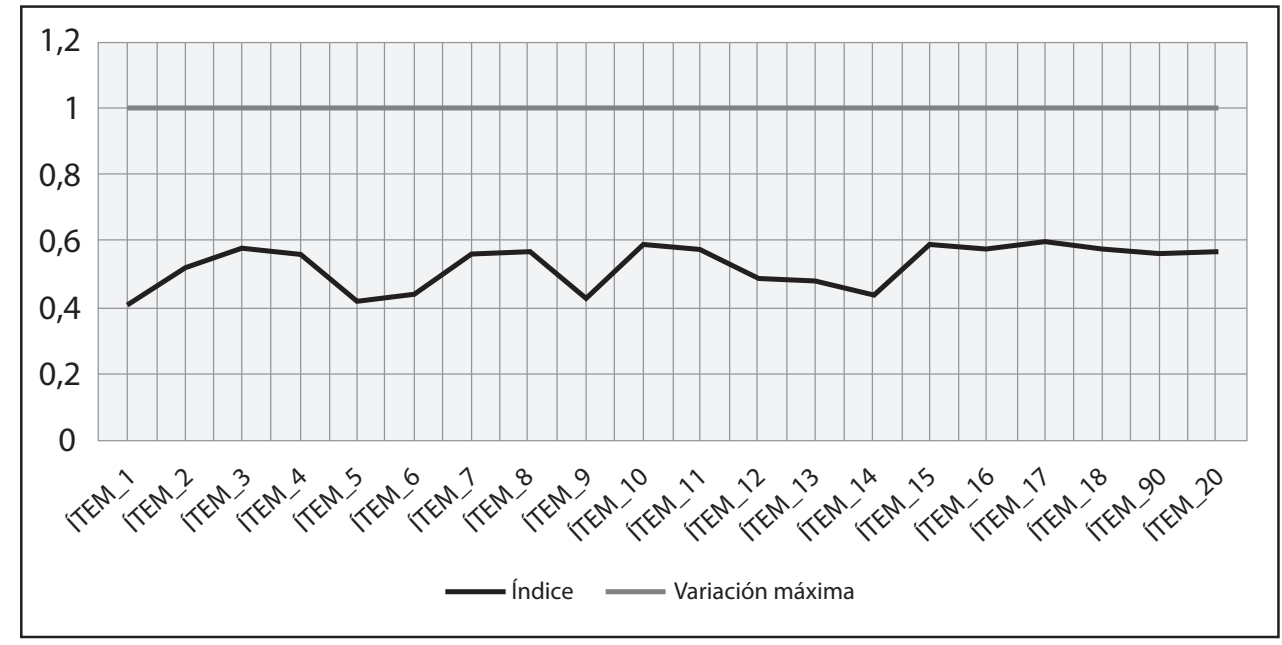

Fuente. Jiménez y Rincón, 2017. 
La tabla 12 y la figura 8 describen el comportamiento de cada dimensión y relación a la escala/índice que se ha tomado de referencia para el análisis, muestra 3 dimensiones en un nivel medio «aceptablemente significativo» $y$ una dimensión en un nivel "poco significativo", la dimensión con menor índice es la social, que tiene un peso de participación del $48 \%$, lo que muestra unos resultados pesimistas frente a la disposición de la pymes de Santa Marta frente al post-acuerdo. En términos generales, la variable disposición de la pymes para el post-acuerdo tiene un índice de 0.53 y se encuentra en un nivel medio «aceptablemente significativo" pero en su cuartil más bajo. Lo que sugiere la necesidad en la construcción de un escenario de mayor divulgación de la política que refrendan los acuerdos del proceso de paz con las FARC en los sectores productivos de la ciudad de Santa Marta. Los resultados de esta variable indican el poco interés y afectación que tiene el contexto social actual del país en las pymes de Santa Marta.

Tabla 12. Niveles de significancia de dimensiones de la variable 2: disposición de pymes para el postacuerdo

\begin{tabular}{|l|c|c|c|}
\hline Dimensiones & índice & Nivel de significancia & Cuartil \\
\hline Dimensión social & 0.49 & P_S & 4 \\
\hline Dimensión económica & 0.54 & A_S & 1 \\
\hline Dimensión ambiental & 0.52 & A_S & 1 \\
\hline Participación social & 0.57 & A_S & 1 \\
\hline VARIABLE & 0.53 & A_S & 1 \\
\hline
\end{tabular}

Fuente. Jiménez y Rincón, 2017.

Figura 8. Niveles de significancia de dimensiones de la variable 2: disposición de pymes para el postacuerdo

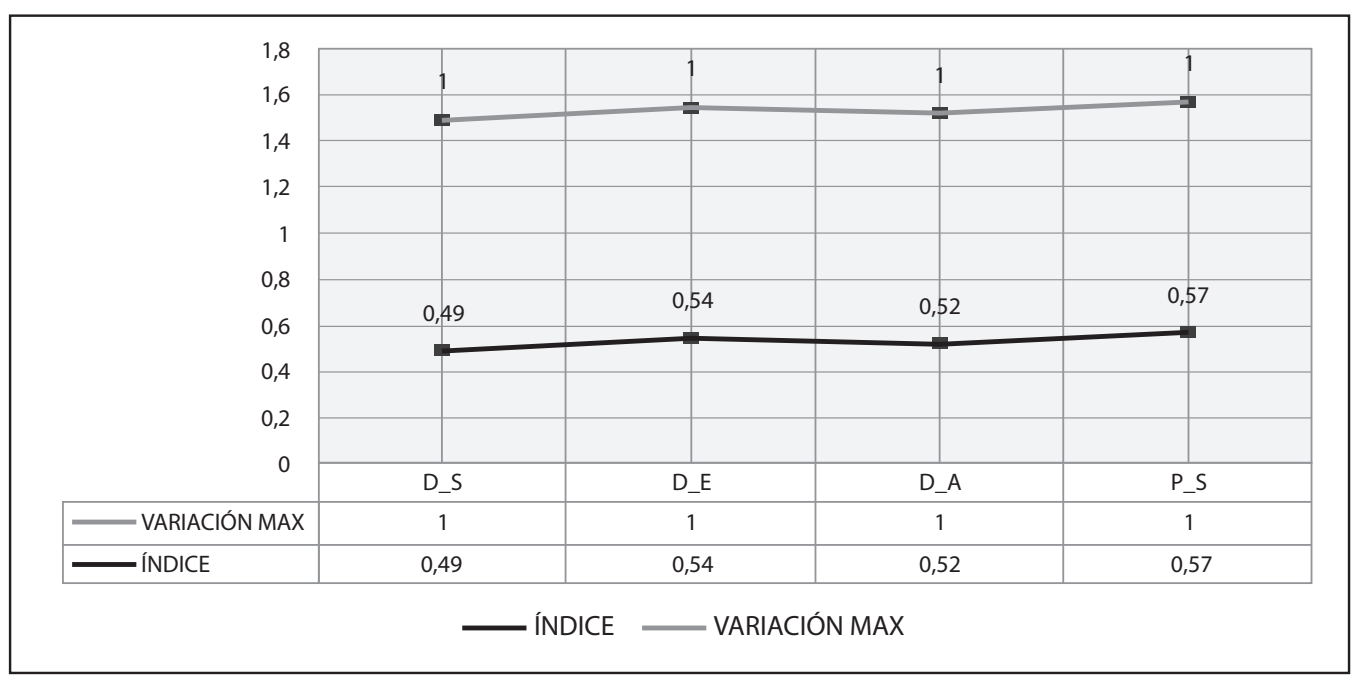

Fuente. Jiménez y Rincón, 2017. 


\subsection{Resultados fase 3}

En la fase final se probó la hipótesis de trabajo midiendo el nivel de correlación de la variable nivel de liderazgo, frente a la variable disposición de la pymes para el post-acuerdo por medio de prueba de hipótesis chi-cuadrado, teniendo como formulación de la hipótesis:

- $\mathrm{H}_{\mathrm{o}} \rightarrow \mathrm{X}_{\mathrm{nb}}(\mathrm{r}) \mathrm{X}_{\mathrm{na}}$-existe una relación dependiente entre el nivel de liderazgo y el índice de disposición de las pymes de Santa Marta para el post-acuerdo-.

- $\mathrm{H}_{1} \rightarrow \mathrm{X}_{\mathrm{nb}} \neq(\mathrm{r}) \mathrm{X}_{\mathrm{na}}-$ no existe una relación dependiente entre el nivel de liderazgo y el índice de disposición de las pymes de Santa Marta para el post-acuerdo-.

Siendo $\mathrm{H}_{\mathrm{o}}$ la hipótesis nula / $\mathrm{X}_{\mathrm{nb}}$, variable $1 / \mathrm{X}_{\mathrm{na}}$ variable $2 / \mathrm{H}_{1}$ la hipótesis de trabajo / (r) correlación.
La tabla 13 y la figura 9 muestra el valor de chi-cuadrado para nuestra prueba de hipótesis: 36.518, teniendo 12 grados de libertad y al revisar la tabla de distribución chi-cuadrado con un grado de significancia de $0.05 \%$ encontramos que el valor es 21.026, este valor es inferior al coeficiente límite lo que acepta la hipótesis planteada, es decir que sí existe una relación entre los niveles de liderazgo y la disposición de las pymes para el post-acuerdo. Se puede formular que:

$$
\mathrm{H}_{\mathrm{o}} \rightarrow \mathrm{X}_{\mathrm{nb}}(\mathrm{r}) \mathrm{X}_{\mathrm{na}}
$$

- Existe una relación dependiente entre el nivel de liderazgo y el índice de disposición de las pymes de Santa Marta para el post-acuerdo-.

Tabla 13. Coeficiente de chi-cuadrado

\begin{tabular}{|c|c|c|c|}
\hline & Valor & Gl & Sig. asintótica (bilateral) \\
\hline Chi-cuadrado de Pearson & $36,518^{\mathrm{a}}$ & 12 &, 000 \\
\hline Razón de verosimilitudes & 38,993 & 12 &, 000 \\
\hline Asociación lineal por lineal & 1,124 & 1 &, 289 \\
\hline N. ${ }^{\circ}$ de casos válidos & 194 & & \\
\hline
\end{tabular}

a 12 casillas $(57.1 \%)$ tienen una frecuencia esperada inferior a 5 . La frecuencia mínima esperada es. 15 .

Fuente. Jiménez y Rincón, 2017.

La siguiente figura muestra de modo gráfico la ubicación del coeficiente de chi-cuadrado dentro del margen de verosimilitud en relación con el resultado de la tabla de grados de libertad, la cual muestra estar dentro del rango de aceptación de prueba de hipótesis. 
Figura 9. Distribución de chi-cuadrado

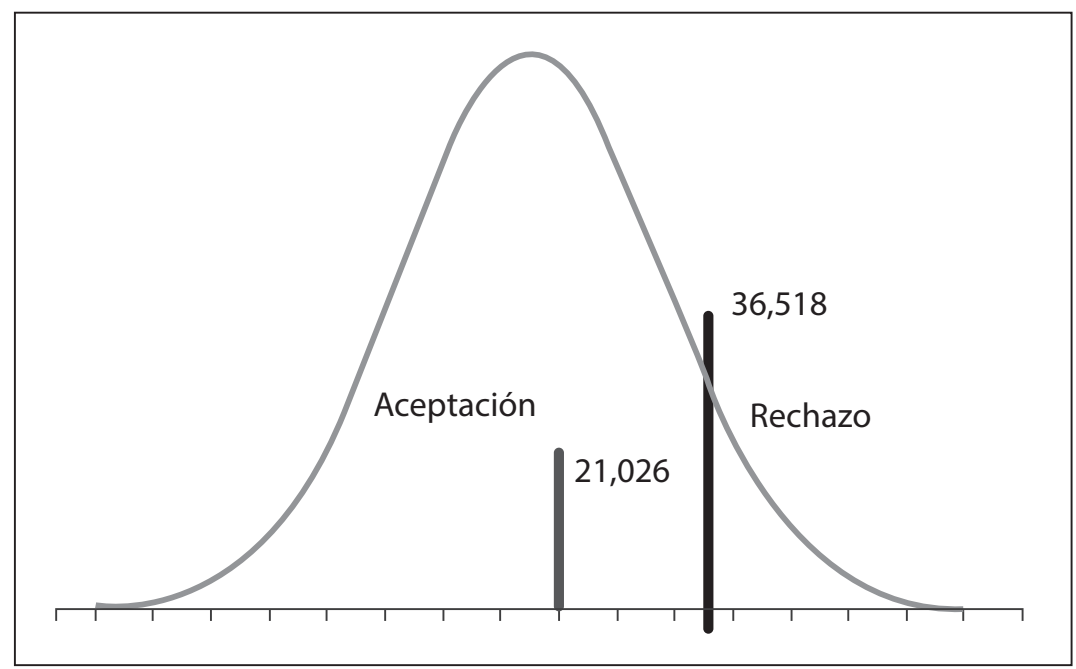

Fuente. Jiménez y Rincón, 2017.

\section{Conclusiones}

C omo primera conclusión, se puede afirmar que la mayoría de las organizaciones tienen un perfil de liderazgo democrático con amplia participación de los grupos de trabajo, lo que posibilita la discusión de temas o tendencias propias del contexto que afectan a las organizaciones, lo que puede dar lugar a una mayor disposición a la hora de incorporar procesos asociados al post-acuerdo.

Como segunda conclusión, se encontró que el índice de disposición de las pymes es aceptablemente significativo en el cuartil más bajo del nivel, lo que significa que existe un desinterés por los temas que están asociados al post-acuerdo, lo que representaría una incorporación casi nula a temas asociados a empleabilidad de población reinsertada, en condiciones de discapacidad, poca participación en temas comunitarios y un conocimiento superficial sobre los acuerdos de paz.
Una tercera conclusión es que al cruzar las dos variables de estudio, se encontró que existe una interdependencia entre los perfiles de liderazgo y la disposición de la pymes para acciones asociadas al post-acuerdo. Esto implica que debe haber mayor interés por parte del Estado para concientizar a las organizaciones del papel que deben jugar en la construcción de la paz.

En el contexto de nuestra actual coyuntura política, en medio de un escenario de post-acuerdo y en etapa de regulación de aplicación de los acuerdos, es necesario acusar un rol con mayor participación del sector empresarial, un rol determinado desde el liderazgo que transforma no solo en el ámbito de la empresa, sino además, las necesidades de su entorno aminorando las brechas e insuficiencias, morales, económicas y sociales de nuestros semejantes (Barradas, 2013). 
Para Gandica (2008), la responsabilidad en términos morales es exigible cuando las decisiones que toman los individuos dentro de las organizaciones afectan a otros individuos o una comunidad en particular. Lo que se afirma o se indica como decisión se convierte en unidad de referencia. La acción social y las actividades de responsabilidad desde las organizaciones pueden definirse como un conjunto de prácticas que las empresas realizan para mejorar las relaciones con sus grupos de interés (Agüero, Carbonell y Martínez, 2006). La RSE es una nueva tendencia; la cuestión es saber si es una tendencia cada vez más consolidada y más coherente por los líderes empresariales o gubernamentales (Barradas, 2013).

De acuerdo con la visión más amplia de la RSE, las empresas son una parte integral de la sociedad en la que estas operan. Sus acciones afectan a la sociedad en general, y por lo tanto son consideradas responsables de sus impactos en la sociedad en su conjunto. Las empresas deben servir de manera constructiva a las necesidades de la sociedad, a satisfacción de esta (Jiménez, 2016). Este contexto requiere un liderazgo con compromiso social que proceda de manera responsable más allá de las actuaciones meramente funcionales a favor de la organización, más allá del sentido tradicional que para la realidad del Caribe colombiano no es suficiente. Los grupos de interés cada vez exigen un mayor compromiso social.

No basta solo con evitar perjuicios en el espectro circundante a las operaciones de las organizaciones, o simples manifestaciones filantrópicas, o donativos de caridad que se requieren para una acción social intrínseca a los líderes empresariales. Para Jarrin (2008), el líder de hoy debe entenderse desde el ámbito moral, como aquel que es responsable de un modelo económico sustentable, y la sustentabilidad media por tres pilares esenciales: lo económico, lo medio ambiental y lo social. Por ello se debe pensar en la necesidad de un "líder social» capaz de extender los compromisos sociales de la organización a la comunidad circundante y su contexto ascensional.

La empresa moderna asume un reto histórico en la construcción de sentido de los procesos de cohesión social, para ello el concepto de RSE se erige como un instrumento de tránsito que nos acerca a la construcción de una sociedad con más equidad y justicia (Jiménez y Pérez, 2016).

\section{Referencias}

Acosta, C. (2015). Relación entre el liderazgo transformacional y la esperanza. Estudio de 100 líderes empresariales chilenos. Visión de futuro, 3858.

Adimen. (25 de octubre de 2016). Calculadora de muestras. Recuperado de http://www.adimen.es/ calculadora-muestras.aspx

Agüero, A., Carbonell, M., y Martínez, J. (2006). Los stakeholders y la acción social de la empresa. Madrid: Jurídicas y Sociales.
Barradas, G. (2013). Del liderazgo visionario hacia la Responsabilidad Social Corporativa. Visión gerencial, 258-280.

Bennis, N. (2001). Líderes, estrategias para un liderazgo eficaz. Barcelona: Paidós.

Cortázar, J. (2004). Sobre la gerencia social: campo de conocimiento y acción. Washington, D. C.: Instituto Interamericano para el Desarrollo Social. 
Fernández, J., y Puentes, A. (2014). La noción de campo en Kurt Lewin y Pierre Bourdieu: un análisis comparativo. Reis. Revista Española de Investigaciones, 33-53.

Gandica, G. (2008). Responsabilidad Social Empresarial. Conciencia Activa, 69-71.

Goleman, D. (2015). Liderazgo, el poder de la inteligencia emocional. Barcelona: Ediciones B.

Goleman, D. (2016). Cómo ser un líder. Barcelona: Ediciones B.

Hernández, F. (2006). Metodología de la investigación científica. México: McGraw-Hill.

Hesselbein, F. (2010). De líder a líder. Buenos Aires: Ediciones Granica S. A.

Jarrin, A. (2008). De la revolución industrial a la evolución empresarial. Caracas: Creating C. A.

Jiménez, D., y Pérez, L. (2016). La representación simbólica de la RSE. Revista Investigación e Innovación en Ingeniería, 24-39.
Jiménez, D. (2016). Prácticas de RSE: un estudio de casos. Sotavento, 94-110.

Kliksberg, B. (2009). Más ética, más desarrollo, (17. ${ }^{\mathrm{a}}$ ed.). Buenos Aires: Editorial Temas.

Lewin, K. (1991). Epistemología comparada. Madrid: Tecnos.

Ospina, S. (2006). Gobernanza y liderazgos para el cambio social. Revista del CLAD Reforma y Democracia, (35), 1-17.

Pírela, L., Camacho, H., y Sánchez, M. (2004). Enfoque epistemológico del liderazgo transformacional. Omnia, 6-21.

Radrigán R., M., y Dávila R., A., y Penaglia V., F. (2012). Gestión y liderazgos en los emprendimientos sociales: el caso del sector no lucrativo chileno. POLIS, Revista Latinoamericana, 11(32) . 